\title{
Stochastic Volatility Jump-Diffusion Model for Option Pricing
}

\author{
Nonthiya Makate, Pairote Sattayatham \\ School of Mathematics, Suranaree University of Technology, Nakhon Ratchasima, Thailand \\ E-mail: nonthiyam@hotmail.com,pairote@sut.ac.th
}

Received July 26, 2011; revised September 2, 2011; accepted September 15, 2011

\begin{abstract}
An alternative option pricing model is proposed, in which the asset prices follow the jump-diffusion model with square root stochastic volatility. The stochastic volatility follows the jump-diffusion with square root and mean reverting. We find a formulation for the European-style option in terms of characteristic functions of tail probabilities.
\end{abstract}

Keywords: Jump-Diffusion Model, Stochastic Volatility, Characteristic Function, Option Pricing

\section{Introduction}

Let $(\Omega, \mathcal{F}, P)$ be a probability space with filtration $\mathcal{F}=\left(\mathcal{F}_{t}\right)_{0 \leq t \leq T}$. All processes that we shall consider in this section will be defined in this space. An asset price model with stochastic volatility has been defined by Heston [1] which has the following dynamics:

$$
\begin{gathered}
\mathrm{d} S_{t}=S_{t}\left(\mu \mathrm{d} t+\sqrt{v_{t}} \mathrm{~d} W_{t}^{S}\right), \\
\mathrm{d} v_{t}=\kappa\left(\theta-v_{t}\right) \mathrm{d} t+\sigma \sqrt{v_{t}} \mathrm{~d} W_{t}^{v},
\end{gathered}
$$

where $S_{t}$ is the asset price, $\mu \in \mathfrak{R}$ is the rate of return of the asset, $v_{t}$ is the volatility of asset returns, $\kappa>0$ is a mean-reverting rate, $\theta \in \mathfrak{R}$ is the long term variance, $\sigma>0$ is the volatility of volatility, $W_{t}^{S}$ and $W_{t}^{v}$ are standard Brownian motions corresponding to the processes $S_{t}$ and $v_{t}$, respectively, with constant correlation $\rho$. In 1996, Bate [2] introduced the jump-diffusion stochastic volatility model by adding log normal jump $Y_{t}$ to the Heston stochastic volatility model. In the original formulation of Bate, the model has the following form:

$$
\begin{gathered}
\mathrm{d} S_{t}=S_{t}\left(\mu \mathrm{d} t+\sqrt{v_{t}} d W_{t}^{S}\right)+S_{t-} Y_{t} \mathrm{~d} N_{t}^{S}, \\
\mathrm{~d} v_{t}=\kappa\left(\theta-v_{t}\right) \mathrm{d} t+\sigma \sqrt{v_{t}} \mathrm{~d} W_{t}^{v},
\end{gathered}
$$

where $N_{t}^{S}$ is the Poisson process which corresponds to the underlying asset $S_{t}, Y_{t}$ is the jump size of asset price return with $\log$ normal distribution and $S_{t-}$ means that there is a jump the value of the process before the jump is used on the left-hand side of the formula. Moreover, in 2003, Eraker Johannes and Polson [3] extended Bate's work by incorporating jumps in volatility and their model is given by

$$
\begin{gathered}
\mathrm{d} S_{t}=S_{t}\left(\mu \mathrm{d} t+\sqrt{v_{t}} \mathrm{~d} W_{t}^{S}\right)+S_{t-} Y_{t} \mathrm{~d} N_{t}^{S}, \\
\mathrm{~d} v_{t}=\kappa\left(\theta-v_{t}\right) \mathrm{d} t+\sigma \sqrt{v_{t}} \mathrm{~d} W_{t}^{v}+Z_{v} \mathrm{~d} N_{t}^{v} .
\end{gathered}
$$

Eraker et al. [3] developed a likelihood-based estimation strategy and provided estimates of parameters, spot volatility, jump times, and jump sizes using S\&P 500 and Nasdaq 100 index returns. Moreover, they examined the volatility structure of the S\&P and Nasdaq indices and indicated that models with jumps in volatility are preferred over those without jumps in volatility. But they did not provide a closed-form formula for the price of a European call option.

In this paper, we would like to consider the problem of finding a closed-form formula for a European call option where the underlying asset and volatility follow the Model (3). This formula will be useful for option pricing rather than an estimation of it as appeared in Eraker's work.

The rest of the paper is organized as follows. In Section 2, we briefly discuss the model descriptions for the option pricing. The relationship between stochastic differential equations and partial differential equations for the jump-diffusion process with jump stochastic volatility is presented in Section 3. Finally, a closed-form formula for a European call option in terms of characteristic functions is presented.

\section{Model Descriptions}

It is assumed that a risk-neutral probability measure $\mathcal{M}$ 
exists, the asset price $S_{t}$ under this measure follows a jumpdiffusion process, and the volatility $v_{t}$ follows a pure mean reverting and square root diffusion process with jump, i.e. our models are governed by the following dynamics:

$$
\begin{aligned}
& \mathrm{d} S_{t}=S_{t}\left(\left(r-\lambda^{S} m\right) \mathrm{d} t+\sqrt{v_{t}} \mathrm{~d} W_{t}^{S}\right)+S_{t-} Y_{t} \mathrm{~d} N_{t}^{S}, \\
& \mathrm{~d} v_{t}=\kappa\left(\theta-v_{t}\right) \mathrm{d} t+\sigma \sqrt{v_{t}} \mathrm{~d} W_{t}^{v}+Z_{t} \mathrm{~d} N_{t}^{v},
\end{aligned}
$$

where $S_{t}, v_{t}, \kappa, \theta, \sigma, W_{t}^{S}$ and $W_{t}^{v}$ are defined as in Bate's model, $r$ is the risk-free interest rate, $N_{t}^{S}$ and $N_{t}^{v}$ are independent Poisson processes with constant intensities $\lambda^{S}$ and $\lambda^{v}$ respectively. $Y_{t}$ is the jump size of the asset price return with density $\phi_{Y}(y)$ and $E\left[Y_{t}\right]:=m<\infty$ and $Z_{t}$ is the jump size of the volatility with density $\phi_{Z}(z)$. Moreover, we assume that the jump processes $N_{t}^{S}$ and $N_{t}^{v}$ are independent of standard Brownian motions $W_{t}^{S}$ and $W_{t}^{v}$.

\section{Partial Integro-Differential Equations}

Consider the process $\boldsymbol{X}_{t}=\left(X_{t}^{(1)}, X_{t}^{(2)}\right)$ where $X_{t}^{(1)}$ and $X_{t}^{(2)}$ are processes in $\Re$ and satisfy the following equations:

$$
\begin{aligned}
\mathrm{d} X_{t}^{(1)}= & f_{1}\left(X_{t}^{(1)}, X_{t}^{(2)}, t\right) \mathrm{d} t+g_{1}\left(X_{t}^{(1)}, X_{t}^{(2)}, t\right) \mathrm{d} W_{t}^{(1)} \\
& +X_{t-}^{(1)} Y_{t} \mathrm{~d} N_{t}^{(1)} \\
\mathrm{d} X_{t}^{(2)}= & f_{2}\left(X_{t}^{(1)}, X_{t}^{(2)}, t\right) \mathrm{d} t+g_{2}\left(X_{t}^{(1)}, X_{t}^{(2)}, t\right) \mathrm{d} W_{t}^{(2)} \\
& +Z_{t} \mathrm{~d} N_{t}^{(2)}
\end{aligned}
$$

where $f_{1}, g_{1}, f_{2}$ and $g_{2}$ are all continuously differentiable, $W_{t}^{(1)}$ and $W_{t}^{(2)}$ are standard Brownian motions with $\operatorname{Corr}\left[\mathrm{d} W_{t}^{(1)}, \mathrm{d} W_{t}^{(2)}\right]=\rho, \quad N_{t}^{(1)}$ and $N_{t}^{(2)}$ are independent Poisson processes with constant intensities $\lambda^{(1)}$ and $\lambda^{(2)}$ respectively.

Since every compound Poisson process can be represented as an integral form of a Poisson random measure [4] then the last term on the right hand side of (5) can be written as follows:

$$
\begin{gathered}
\int_{0}^{t} X_{s-}^{(1)} Y_{s} \mathrm{~d} N_{s}^{(1)}=\sum_{n=1}^{N_{t}^{(1)}} X_{n-}^{(1)} Y_{n}=\int_{0}^{t} \int_{\Re} X_{s-}^{(1)} q J_{Q}(\mathrm{~d} s \mathrm{~d} q), \\
\int_{0}^{t} Z_{s} \mathrm{~d} N_{s}^{(2)}=\sum_{n=1}^{N_{t}^{(2)}} Z_{n}=\int_{0}^{t} \int_{\Re} r J_{R}(\mathrm{~d} s \mathrm{~d} r),
\end{gathered}
$$

where $Y_{n}$ are i.i.d. random variables with density $\phi_{Y}(y)$ and $J_{1}(i)$ is a Poisson random measure of the process $Q_{t}=\sum Y_{n}$ with intensity measure $\lambda^{(1)} \phi_{Y}(\mathrm{~d} q) \mathrm{d} t, Z_{n}$ are i.i.d. random variables with density $\phi_{Z}(z)$, and $J_{R}$ is a
Poisson random measure of the process $R_{t}=\sum_{n=1}^{N_{t}^{(2)}} Z_{n}$ with intensity measure $\lambda^{(2)} \phi_{Z}(\mathrm{~d} r) \mathrm{d} t$.

Let $U\left(x_{1}, x_{2}\right)$ be a bounded real-valued function and twice continuously differentiable with respect to $x_{1}$ and $x_{2}$ and

$$
u\left(x_{1}, x_{2}, t\right)=E\left[U\left(X_{T}^{(1)}, X_{T}^{(2)}\right) \mid X_{t}^{(1)}=x_{1}, X_{t}^{(2)}=x_{2}\right](6)
$$

By the two dimensional Dynkin formula [5], $u$ is a solution of the partial integro-differential equation (PIDE)

$$
\begin{aligned}
0= & \frac{\partial u\left(x_{1}, x_{2}, t\right)}{\partial t}+\overline{\mathcal{A}} u\left(x_{1}, x_{2}, t\right) \\
& +\lambda^{(1)} \int_{\Re}\left[u\left(x_{1}+y, x_{2}, t\right)-u\left(x_{1}, x_{2}, t\right)\right] \phi_{Y}(y) \mathrm{d} y \\
& +\lambda^{(2)} \int_{\Re}\left[u\left(x_{1}, x_{2}+z, t\right)-u\left(x_{1}, x_{2}, t\right)\right] \phi_{Z}(z) \mathrm{d} z
\end{aligned}
$$

subject to the final condition $u\left(x_{1}, x_{2}, T\right)=U\left(x_{1}, x_{2}\right)$. The notation $\overline{\mathcal{A}}$ is defined by

$$
\begin{aligned}
\bar{A} u\left(x_{1}, x_{2}, t\right)= & f_{1} \frac{\partial u\left(x_{1}, x_{2}, t\right)}{\partial x_{1}}+f_{2} \frac{\partial u\left(x_{1}, x_{2}, t\right)}{\partial x_{2}} \\
& +\frac{1}{2} g_{1}^{2} \frac{\partial^{2} u\left(x_{1}, x_{2}, t\right)}{\partial x_{1}^{2}}+\rho g_{1} g_{2} \frac{\partial^{2} u\left(x_{1}, x_{2}, t\right)}{\partial x_{1} \partial x_{2}} \\
& +\frac{1}{2} g_{2}^{2} \frac{\partial^{2} u\left(x_{1}, x_{2}, t\right)}{\partial x_{2}^{2}}
\end{aligned}
$$

\section{A Closed-Form Formula for the Price of a European Call Option}

Let $C$ denote the price at time $t$ of a European style call option on the current price of the underlying asset $S_{t}$ with strike price $K$ and expiration time $T$.

The terminal payoff of a European call option on the underlying stock $S_{t}$ with strike price $K$ is

$$
\max \left(S_{T}-K, 0\right) .
$$

This means that the holder will exercise his right only if $S_{T}>K$ and then his gain is $S_{T}-K$. Otherwise, if $S_{T} \leq K$, then the holder will buy the underlying asset from the market and the value of the option is zero.

Assuming the risk-free interest rate $r$ is constant over the lifetime of the option, the price of the European call at time $t$ is equal to the discounted conditional expected payoff 


$$
\begin{aligned}
& C\left(S_{t}, v_{t}, t ; K, T\right) \\
= & e^{-r(T-t)} E_{\mathcal{M}}\left[\max \left(S_{T}-K, 0\right) \mid S_{t}, v_{t}\right] \\
= & e^{-r(T-t)}\left(\int_{K}^{\infty} S_{T} P_{\mathcal{M}}\left(S_{T} \mid S_{t}, v_{t}\right) \mathrm{d} S_{T}-K \int_{K}^{\infty} P_{\mathcal{M}}\left(S_{T} \mid S_{t}, v_{t}\right) \mathrm{d} S_{T}\right) \\
= & S_{t}\left(\frac{1}{\mathrm{e}^{r(T-t)} S_{t}} \int_{K}^{\infty} S_{T} P_{\mathcal{M}}\left(S_{T} \mid S_{t}, v_{t}\right) \mathrm{d} S_{T}\right) \\
& -K e^{-r(T-t)} \int_{K}^{\infty} P_{\mathcal{M}}\left(\left(S_{T} \mid S_{t}, v_{t}\right)\right) \mathrm{d} S_{T} \\
= & S_{t}\left(\frac{1}{E_{\mathcal{M}}\left[S_{T} \mid S_{t}, v_{t}\right]} \int_{K}^{\infty} S_{T} P_{\mathcal{M}}\left(S_{T} \mid S_{t}, v_{t}\right) \mathrm{d} S_{T}\right) \\
& -K \mathrm{e}^{-r(T-t)} \int_{K}^{\infty} P_{\mathcal{M}}\left(S_{T} \mid S_{t}, v_{t}\right) \mathrm{d} S_{T} \\
= & S_{t} P_{1}\left(S_{t}, v_{t}, t ; K, T\right)-K e^{-r(T-t)} P_{2}\left(S_{t}, v_{t}, t ; K, T\right)
\end{aligned}
$$

where $E_{\mathcal{M}}$ is the expectation with respect to the riskneutral probability measure, $P_{\mathcal{M}}\left(S_{T} \mid S_{t}, v_{t}\right)$ is the corresponding conditional density given $\left(S_{t}, v_{t}\right)$ and

$$
P_{1}\left(S_{t}, v_{t}, t ; K, T\right)=\left(\int_{K}^{\infty} S_{T} P_{\mathcal{M}}\left(S_{T} \mid S_{t}, v_{t}\right) \mathrm{d} S_{T}\right) / E_{\mathcal{M}}\left[S_{T} \mid S_{t}, v_{t}\right]
$$

Note that $P_{1}$ is the risk-neutral probability that $S_{T}>K$ (since the integrand is nonnegative and the integral over $[0, \infty)$ is one), and finally that

$$
\begin{aligned}
P_{2}\left(S_{t}, v_{t}, t ; K, T\right) & =\int_{K}^{\infty} P_{\mathcal{M}}\left(S_{T} \mid S_{t}, v_{t}\right) \mathrm{d} S_{T} \\
& =\operatorname{Prob}\left(S_{T}>K \mid S_{t}, v_{t}\right)
\end{aligned}
$$

is the risk-neutral in-the-money probability. Moreover,

$$
E_{\mathcal{M}}\left[S_{T} \mid S_{t}, v_{t}\right]=\mathrm{e}^{r(T-t)} S_{t} \text { for } t \geq 0 .
$$

Assume that the asset price $S_{t}$ and the volatility $v_{t}$ satisfy (4), we would like to compute the price of a European call option with strike price $K$ and maturity $T$. To do this, we make a change of variable from $S_{t}$ to $L_{t}=\ln S_{t}$, i.e. where $S_{t}$ satisfies (4) and its inverse $S_{t}=e^{L_{t}}$. Denote $k=\ln K$ the logarithm of the strike price. By the jump-diffusion chain rule, $\ln S_{t}$ satisfies the SDE

$$
d \ln S_{t}=\left(r-\lambda^{S} m-\frac{v_{t}}{2}\right) \mathrm{d} t+\sqrt{v_{t}} \mathrm{~d} W_{t}^{S}+\ln \left(1+Y_{t}\right) \mathrm{d} N_{t}^{S}(9)
$$

Applying the two-dimensional Dynkin formula [5] for the price dynamics (9) and volatility $v_{t}$ in system (4), we obtain the value of a European-style option, as a function of the stock log-return $L_{t}$ denoted by

$$
\begin{aligned}
\tilde{C}\left(L_{t}, v_{t}, t ; k, T\right) & \equiv C\left(e^{L_{t}}, v_{t}, t ; e^{k}, T\right) \\
& =C\left(e^{\ln S_{t}}, v_{t}, t ; e^{\ln K}, T\right) \\
& =C\left(S_{t}, v_{t}, t ; K, T\right),
\end{aligned}
$$

i.e.,

$$
\tilde{C}(l, v, t ; k, T)=e^{-r(T-t)} E_{\mathcal{M}}\left[\max \left(e^{L_{T}}-K, 0\right) \mid L_{t}=l, v_{t}=v\right]
$$

and satisfies the following PIDE:

$$
\begin{aligned}
0= & \frac{\partial \tilde{C}}{\partial t}+\overline{\mathcal{A}}[\tilde{C}](l, v, t ; k, T) \\
& +\lambda^{S} \int_{\Re}[\tilde{C}(l+y, v, t ; k, T)-\tilde{C}(l, v, t ; k, T)] \phi_{Y}(y) \mathrm{d} y \\
& +\lambda^{v} \int_{\Re}[\tilde{C}(l, v+z, t ; k, T)-\tilde{C}(l, v, t ; k, T)] \phi_{Z}(z) \mathrm{d} z
\end{aligned}
$$

Here the operator $\overline{\mathcal{A}}$ as in (7) is defined by

$$
\begin{aligned}
\overline{\mathcal{A}}[\tilde{C}](l, v, t ; k, T)= & \left(r-\lambda^{S} m-\frac{1}{2} v\right) \frac{\partial \tilde{C}}{\partial l}+\kappa(\theta-v) \frac{\partial \tilde{C}}{\partial v} \\
& +\frac{1}{2} v \frac{\partial^{2} \tilde{C}}{\partial l^{2}}+\rho \sigma v \frac{\partial^{2} \tilde{C}}{\partial l \partial v} \\
& +\frac{1}{2} \sigma^{2} v \frac{\partial^{2} \tilde{C}}{\partial v^{2}}-r \tilde{C}
\end{aligned}
$$

In the current state variable, the last line of (8) becomes

$$
\tilde{C}(l, v, t ; k, T)=e^{l} \tilde{P}_{1}(l, v, t ; k, T)-e^{k-r(T-t)} \tilde{P}_{2}(l, v, t ; k, T)
$$

where $\tilde{P}_{j}(l, v, t ; k, T):=P_{j}\left(e^{l}, v, t ; e^{k}, T\right), j=1,2$.

The following lemma shows the relationship between $\tilde{P}_{1}$ and $\tilde{P}_{2}$ in the option value of (11).

Lemma 1 The functions $\tilde{P}_{1}$ and $\tilde{P}_{2}$ in the option value of (11) satisfy the following PIDEs

$$
\begin{aligned}
0= & \frac{\partial \tilde{P}_{1}}{\partial t}+\mathcal{A}\left[\tilde{P}_{1}\right](l, v, t ; k, T)+v \frac{\partial \tilde{P}_{1}}{\partial l} \\
& +\rho \sigma v \frac{\partial \tilde{P}_{1}}{\partial v}+\left(r-\lambda^{S} m\right) \tilde{P}_{1} \\
& +\lambda^{S} \int_{\Re}\left[\left(e^{y}-1\right) \tilde{P}_{1}(l+y, v, t: k, T)\right] \phi_{Y}(y) \mathrm{d} y
\end{aligned}
$$

and subject to the boundary condition at expiration time $t=T$;

$$
\tilde{P}_{1}(l, v, T ; k, T)=1_{l>k}
$$

moreover, $\tilde{P}_{2}$ satisfies the equation

$$
0=\frac{\partial \tilde{P}_{2}}{\partial t}+\mathcal{A}\left[\tilde{P}_{2}\right](l, v, t ; k, T)+r \tilde{P}_{2},
$$


and subject to the boundary condition at expiration time $t=T$;

$$
\tilde{P}_{2}(l, v, T ; k, T)=1_{l>k},
$$

The operator $\mathcal{A}$ is defined by

$$
\begin{aligned}
& \mathcal{A}[f](l, v, t ; k, T) \\
:= & \left(r-\lambda^{S} m-\frac{1}{2} v\right) \frac{\partial f}{\partial l}+\kappa(\theta-v) \frac{\partial f}{\partial v}+\frac{1}{2} v \frac{\partial^{2} f}{\partial l^{2}} \\
& +\rho \sigma v \frac{\partial^{2} f}{\partial l \partial v}+\frac{1}{2} \sigma^{2} v \frac{\partial^{2} f}{\partial v^{2}}-r f \\
& +\lambda^{S} \int_{\Re}[f(l+y, v, t ; k, T)-f(l, v, t ; k, T)] \phi_{Y}(y) \mathrm{d} y \\
& +\lambda^{v} \int_{\Re}[f(l, v+z, t ; k, T)-f(l, v, T ; k, T)] \phi_{Z}(z) \mathrm{d} z .
\end{aligned}
$$

Note that $1_{l>k}=1$ if $l>k$ and otherwise $1_{l>k}=0$.

The following lemma shows how to calculate the functions $\tilde{P}_{1}$ and $\tilde{P}_{2}$ as they appeared in Lemma 1 .

Lemma 2 The functions $\tilde{P}_{1}$ and $\tilde{P}_{2}$ can be calculated by the inverse Fourier transforms of the characteristic function, i.e.

$$
\tilde{P}_{j}(l, v, t ; k, T)=\frac{1}{2}+\frac{1}{\pi} \int_{0^{+}}^{+\infty} \operatorname{Re}\left[\frac{e^{-i x k} f_{j}(l, v, t ; k, T)}{i x}\right] \mathrm{d} x,
$$

for $j=1,2$ with $\operatorname{Re}[\cdot]$ denoting the real component of a complex number. By letting $\tau=T-t$.

1) The characteristic function $f_{1}$ is given by

$$
f_{1}(l, v, t ; x, t+\tau)=\exp \left(g_{1}(\tau)+v h_{1}(\tau)+\mathrm{ixl}\right),
$$

where

$$
\begin{gathered}
h_{1}(\tau)=\frac{\left(\eta_{1}^{2}-\Delta_{1}^{2}\right)\left(e^{\Delta_{1} \tau}-1\right)}{\sigma^{2}\left(\eta_{1}+\Delta_{1}-\left(\eta_{1}-\Delta_{1}\right) e^{\Delta_{1} \tau}\right)} \\
g_{1}(\tau)=\left(\left(r-\lambda^{S} m\right) i x-\lambda^{S} m\right) \tau \\
-\frac{\kappa \theta}{\sigma^{2}}\left(2 \ln \left(1-\frac{\left(\Delta_{1}+\eta_{1}\right)\left(1-e^{-\Delta_{1} \tau}\right)}{2 \Delta_{1}}\right)+\left(\Delta_{1}+\eta_{1}\right) \tau\right) \\
+\lambda^{S} \tau \int_{-\infty}^{\infty}\left(e^{(i x+1) y}-1\right) \phi_{Y}(y) \mathrm{d} y \\
+\lambda^{v} \tau \int_{-\infty}^{\infty}\left(e^{z h_{1}(\tau)}-1\right) \phi_{Z}(z) \mathrm{d} z \\
\eta_{1}=\rho \sigma(i x+1)-\kappa \\
\Delta_{1}=\sqrt{\eta_{1}^{2}-\sigma^{2} i x(i x+1)} .
\end{gathered}
$$

and
2) The characteristic function $f_{2}$ is given by

$$
f_{2}(l, v, t ; x, t+\tau)=\exp \left(g_{2}(\tau)+v h_{2}(\tau)+i x l+r \tau\right),
$$$$
\text { where } \quad h_{2}(\tau)=\frac{\left(\eta_{2}^{2}-\Delta_{2}^{2}\right)\left(e^{\Delta_{2} \tau}-1\right)}{\sigma^{2}\left(\eta_{2}+\Delta_{2}-\left(\eta_{2}-\Delta_{2}\right) e^{\Delta_{2} \tau}\right)} \text {, }
$$

$$
g_{2}(\tau)=\left(\left(r-\lambda^{S} m\right) \mathrm{ix}-r\right) \tau
$$$$
-\frac{\kappa \theta}{\sigma^{2}}\left(2 \ln \left(1-\frac{\left(\Delta_{2}+\eta_{2}\right)\left(1-e^{-\Delta_{2} \tau}\right)}{2 \Delta_{2}}\right)+\left(\Delta_{2}+\eta_{2}\right) \tau\right)
$$$$
+\lambda^{S} \tau \int_{-\infty}^{\infty}\left(e^{i x y}-1\right) \phi_{Y}(y) \mathrm{d} y+\lambda^{v} \tau \int_{-\infty}^{\infty}\left(e^{z h_{2}(\tau)}-1\right) \phi_{Z}(z) \mathrm{d} z
$$

$$
\eta_{2}=i \rho \sigma x-\kappa
$$

and

$$
\Delta_{2}=\sqrt{\eta_{2}^{2}-\sigma^{2} i x(i x-1)} .
$$

In summary, we have just proved the following main theorem.

Theorem 3 The value of a European call option of (4) is

$$
\tilde{C}(l, v, t ; k, T)=e^{l} \tilde{P}_{1}(l, v, t ; k, T)-e^{k-r(T-t)} \tilde{P}_{2}(l, v, t ; k, T)
$$

where $\tilde{P}_{1}$ and $\tilde{P}_{2}$ are given in Lemma 2 .

\section{Conclusions}

This paper has proposed asset price dynamics to accommodate both jump-diffusion and jump stochastic volatility. Under this proposed model, an analytical solution is derived for a European call option via the characteristic function.

\section{Acknowledgements}

This research is (partially) supported by The Centre of Excellence in Mathematics, the Commission on Higher Education (CHE).

Address: 272 Rama VI Road, Ratchathewi District, Bangkok, Thailand.

\section{References}

[1] S. L. Heston, "A Close Form Solution for Options with Stochastic Volatility with Applications to Bond and Currency Options," The Review of Financial Studies, Vol. 6, No. 2, 1993, pp. 327-343. doi:10.1093/rfs/6.2.327

[2] D. Bates, "Jump and Stochastic Volatility: Exchange Rate Processes Implicit in Deutsche Mark in Options," Review of Financial Studies, Vol. 9, No. 1, 1996, pp. 69-107. tp://dx.doi.org/10.1093/rfs/9.1.69 
[3] B. Eraker, M. Johannes and N. Polson, "The Impact of Jumps in Volatility and Returns," The Journal of Finance, Vol. 58, No. 3, 2003, pp. 1269-1300. doi:10.1111/1540-6261.00566

[4] R. Cont and P. Tankov, "Financial Modeling with Jump
Processes," CRC Press, Boca Raton, 2004.

[5] F. B. Hanson, "Applied Stochastic Process and Control for Jump Diffusions: Modeling, Analysis and Computation," Society for Industrial and Applied Mathematics, Philadelphia, 2007 


\section{Appendix}

Proof of Lemma 1. We plan to substitute (11) into (10). Firstly, we compute

$$
\begin{aligned}
& \frac{\partial \tilde{C}}{\partial t}=e^{l} \frac{\partial \tilde{P}_{1}}{\partial t}-e^{k-r(T-t)} \frac{\partial \tilde{P}_{2}}{\partial t}-r e^{k-r(T-t)} \tilde{P}_{2} \\
& \frac{\partial \tilde{C}}{\partial l}=e^{l} \frac{\partial \tilde{P}_{1}}{\partial l}+e^{l} \tilde{P}_{1}-e^{k-r(T-t)} \frac{\partial \tilde{P}_{2}}{\partial l} \\
& \frac{\partial \tilde{C}}{\partial v}=e^{l} \frac{\partial \tilde{P}_{1}}{\partial v}-e^{k-r(T-t)} \frac{\partial \tilde{P}_{2}}{\partial v} \\
& \frac{\partial^{2} \tilde{C}}{\partial l^{2}}=e^{l} \frac{\partial^{2} \tilde{P}_{1}}{\partial l^{2}}+2 e^{l} \frac{\partial \tilde{P}_{1}}{\partial l}+e^{l} \tilde{P}_{1}-e^{k-r(T-t)} \frac{\partial^{2} \tilde{P}_{2}}{\partial l^{2}} \\
& \frac{\partial^{2} \tilde{C}}{\partial l \partial v}=e^{l} \frac{\partial^{2} \tilde{P}_{1}}{\partial l \partial v}+e^{l} \frac{\partial \tilde{P}_{1}}{\partial v}-e^{k-r(T-t)} \frac{\partial^{2} \tilde{P}_{2}}{\partial l \partial v} \\
& \frac{\partial^{2} \tilde{C}}{\partial v^{2}}=e^{l} \frac{\partial^{2} \tilde{P}_{1}}{\partial v^{2}}-e^{k-r(T-t)} \frac{\partial^{2} \tilde{P}_{2}}{\partial v^{2}},
\end{aligned}
$$$$
\tilde{C}(l+y, v, t ; k, T)-\tilde{C}(l, v, t ; k, T)
$$$$
=\left[\begin{array}{l}
e^{(l+y)} \tilde{P}_{1}(l+y, v, t ; k, T) \\
-e^{k-r(T-t)} \tilde{P}_{1}(l+y, v, t ; k, T)
\end{array}\right]
$$$$
0=\frac{\partial \tilde{P}_{1}}{\partial t}+\left(r-\lambda^{S} m-\frac{1}{2} v\right)\left(\frac{\partial \tilde{P}_{1}}{\partial l}+\tilde{P}_{1}\right)+\kappa(\theta-v) \frac{\partial \tilde{P}_{1}}{\partial v}+\frac{1}{2} v\left(\frac{\partial^{2} \tilde{P}_{1}}{\partial l^{2}}+2 \frac{\partial \tilde{P}_{1}}{\partial l}+\tilde{P}_{1}\right)
$$$$
+\rho \sigma v\left(\frac{\partial^{2} \tilde{P}_{1}}{\partial l \partial v}+\frac{\partial \tilde{P}_{1}}{\partial v}\right)+\frac{1}{2} \sigma^{2} v \frac{\partial^{2} \tilde{P}_{1}}{\partial \nu^{2}}-r \tilde{P}_{1}+\lambda^{S} \int_{\Re}\left[\left(e^{y}-1\right) \tilde{P}_{1}(l+y, v, t ; k, T)+\tilde{P}_{1}(l+y, v, t ; k, T)-\tilde{P}_{1}(l, v, t ; k, T)\right] \phi_{Y}(y) d y
$$$$
+\lambda^{v} \int_{\Re}\left[\tilde{P}_{1}(l, v+z, t ; k, T)-\tilde{P}_{1}(l, v, t ; k, T)\right] \phi_{Z}(z) \mathrm{d} z
$$

and

$$
\begin{aligned}
\tilde{C} & (l, v+z, t ; k, T)-\tilde{C}(l, v, t ; k, T) \\
= & {\left[e^{l} \tilde{P}_{1}(l, v+z, t ; k, T)-e^{k-r(T-t)} \tilde{P}_{2}(l, v+z, t ; k, T)\right] } \\
& -\left[e^{l} \tilde{P}_{1}(l, v, t ; k, T)-e^{k-r(T-t)} \tilde{P}_{2}(l, v, t ; k, T)\right] \\
= & e^{l}\left[\tilde{P}_{1}(l, v+z, t ; k, T)-\tilde{P}_{1}(l, v, t ; k, T)\right] \\
& -e^{k-r(T-t)}\left[\tilde{P}_{2}(l, v+z, t ; k, T)-\tilde{P}_{2}(l, v, t ; k, T)\right]
\end{aligned}
$$

We substitute all terms above into (10) and separate it by assumed independent terms of $\tilde{P}_{1}$ and $\tilde{P}_{2}$. This gives two PIDEs for the risk-neutralized probability for $\tilde{P}_{j}(l, v, t ; k, T), j=1,2:$ (Equation (15)) subject to the boundary condition at the expiration time $t=T$ according to (12).

By using the notation in (14), PIDE (15) becomes

$$
\begin{aligned}
0=\frac{\partial \tilde{P}_{1}}{\partial t} & +\mathcal{A}\left[\tilde{P}_{1}\right](l, v, t ; k, T)+v \frac{\partial \tilde{P}_{1}}{\partial l} \\
& +\rho \sigma v \frac{\partial \tilde{P}_{1}}{\partial v}+\left(r-\lambda^{S} m\right) \tilde{P}_{1} \\
& +\lambda^{s} \int_{\Re}\left[\left(e^{y}-1\right) \tilde{P}_{1}(l+y, v, t ; k, T)\right] \phi_{Y}(y) d y \\
:=\frac{\partial \tilde{P}_{1}}{\partial t} & +\mathcal{A}_{1}\left[\tilde{P}_{1}\right](l, v, t ; k, T)
\end{aligned}
$$

For $\widetilde{P_{2}}(l, v, t ; k, T)$ :

$$
0=\frac{\partial \tilde{P}_{2}}{\partial t}+r \tilde{P}_{2}+\left(r-\lambda^{S} m-\frac{1}{2} v\right) \frac{\partial \tilde{P}_{2}}{\partial l}+\kappa(\theta-v) \frac{\partial \tilde{P}_{2}}{\partial v}
$$

$$
\begin{aligned}
& +\frac{1}{2} v \frac{\partial^{2} \tilde{P}_{2}}{\partial l^{2}}+\rho \sigma v \frac{\partial^{2} \tilde{P}_{2}}{\partial l \partial v}+\frac{1}{2} \sigma^{2} v \frac{\partial^{2} \tilde{P}_{2}}{\partial v^{2}}-r \tilde{P}_{2} \\
& +\lambda^{s} \int_{\Re}\left[\tilde{P}_{2}(l+y, v, t ; k, T)-\tilde{P}_{2}(l, v, t ; k, T)\right] \phi_{Y}(y) \mathrm{d} y \\
& +\lambda^{v} \int_{\Re}\left[\tilde{P}_{2}(l, v+z, t ; k, T)-\tilde{P}_{2}(l, v, t ; k, T)\right] \phi_{Z}(z) \mathrm{d} z
\end{aligned}
$$

subject to the boundary condition at the expiration time $t=T$ according to (13). Again, by using the notation in (14), PIDE (16) becomes

$$
\begin{gathered}
0=\frac{\partial \tilde{P}_{2}}{\partial t}+\mathcal{A}\left[\tilde{P}_{2}\right](l, v, t ; k, T)+r \tilde{P}_{2} \\
:=\frac{\partial \tilde{P}_{2}}{\partial t}+\mathcal{A}_{2}\left[\tilde{P}_{2}\right](l, v, t ; k, T) .
\end{gathered}
$$


The proof of Lemma 1 is now completed.

For $j=1,2$ the characteristic functions for

$$
\tilde{P}_{j}(l, v, t ; k, T),
$$

with respect to the variable $k$ are defined by

$$
f_{j}(l, v, t ; x, T):=-\int_{-\infty}^{\infty} e^{i x k} \mathrm{~d} \tilde{P}_{j}(l, v, t ; k, T),
$$

with a minus sign to account for the negativity of the measure $\mathrm{d} \tilde{P}_{j}$.

Note that $f_{j}$ also satisfies similar PIDEs

$$
\frac{\partial f_{j}}{\partial t}+\mathcal{A}_{j}\left[f_{j}\right](l, v, t ; x, T)=0,
$$

with the respective boundary conditions

$$
\begin{aligned}
f_{j}(l, v, t ; x, T) & =-\int_{-\infty}^{\infty} e^{i x k} \mathrm{~d} \tilde{P}_{j}(l, v, t ; k, T) \\
& =-\int_{-\infty}^{\infty} e^{i x k}(-\delta(k-l) \mathrm{d} k) \\
& =e^{i x l}
\end{aligned}
$$

since $\mathrm{d} \tilde{P}_{j}(l, v, T ; k, T)=\mathrm{d} 1_{l>k}=\mathrm{d} H(l-k)=-\delta(k-l) \mathrm{d} k$.

\section{Proof of Lemma 2}

1) To solve for the characteristic function explicitly, letting $\tau=T-t$ be the time-to-go, we conjecture that the function $f_{1}$ is given by

$$
f_{1}(l, v, t ; x, t+\tau)=\exp \left(g_{1}(\tau)+v h_{1}(\tau)+i x l\right)
$$

and the boundary condition

$$
g_{1}(0)=0=h_{1}(0) \text {. }
$$

This conjecture exploits the linearity of the coefficient in PIDE (17).

Note that the characteristic function $f_{1}$ always exists.

In order to substitute (18) into (17), firstly we compute

$$
\begin{array}{lc}
\frac{\partial f_{1}}{\partial t}=\left(-g_{1}^{\prime}(\tau)-v h_{1}^{\prime}(\tau)\right) f_{1} & \frac{\partial f_{1}}{\partial l}=i x f_{1} \\
\frac{\partial f_{1}}{\partial v}=h_{1}(\tau) f_{1} & \frac{\partial^{2} f_{1}}{\partial l^{2}}=-x^{2} f_{1} \\
\frac{\partial^{2} f_{1}}{\partial l \partial v}=i x h_{1}(\tau) f_{1} & \frac{\partial^{2} f_{1}}{\partial v^{2}}=h_{1}^{2}(\tau) f_{1} \\
f_{1}(l+y, v, t ; x, t+\tau)-f_{1}(l, v, t ; x, t+\tau) \\
=\left(e^{i x y}-1\right) f_{1}(l, v, t ; x, t+\tau) \\
f_{1}(l, v+z, t ; x, t+\tau)-f_{1}(l, v, t ; x, t+\tau) \\
=\left(e^{z h_{1}(\tau)}-1\right) f_{1}(l, v, t ; x, t+\tau)
\end{array}
$$

and

$$
\begin{aligned}
\left(e^{y}-1\right) f_{1}(l+y, v, t ; x, t+\tau) & =\left(e^{y}-1\right) e^{g_{1}(\tau)+v h_{1}(\tau)+i x(l+y)} \\
& =\left(e^{y}-1\right) e^{i x y} f_{1}(l, v, t ; x, t+\tau)
\end{aligned}
$$

Substituting all the above terms into (17) and after canceling the common factor of $f_{1}$, we get a simplified form as follows:

$$
\begin{aligned}
0= & -g_{1}^{\prime}(\tau)-v h_{1}^{\prime}(\tau)+\left(r-\lambda^{S} m+\frac{1}{2} v\right) i x \\
& +(\kappa(\theta-v)+\rho \sigma v) h_{1}(\tau)-\frac{1}{2} v x^{2} \\
& +\rho \sigma v i x h_{1}(\tau)+\frac{1}{2} \sigma^{2} v h_{1}^{2}(\tau)-\lambda^{S} m \\
& +\lambda^{S} \int_{\Re}\left(e^{(i x+1) y}-1\right) \phi_{Y}(y) \mathrm{d} y \\
& +\lambda^{v} \int_{\Re}\left(e^{z h_{1}(\tau)}-1\right) \phi_{Z}(z) \mathrm{d} z .
\end{aligned}
$$

By separating the order $v$ and ordering the remaining terms, we can reduce it to two ordinary differential equation (ODEs),

$h_{1}^{\prime}(\tau)=\frac{1}{2} \sigma^{2} h_{1}^{2}(\tau)+(\rho \sigma(1+i x)-\kappa) h_{1}(\tau)+\frac{1}{2} i x-\frac{1}{2} x^{2}$

and

$$
\begin{aligned}
g_{1}^{\prime}(\tau)= & \kappa \theta h_{1}(\tau)+\left(r-\lambda^{S} m\right) i x-\lambda^{S} m \\
& +\lambda^{S} \int_{-\infty}^{\infty}\left(e^{(i x+1) y}-1\right) \phi_{Y}(y) \mathrm{d} y \\
& +\lambda^{v} \int_{-\infty}^{\infty}\left(e^{z h_{1}(\tau)}-1\right) \phi_{Z}(z) \mathrm{d} z .
\end{aligned}
$$

Let $\eta_{1}=\rho \sigma(i x+1)-\kappa$ and substitute it into (19). We get

$$
\begin{aligned}
h_{1}^{\prime}(\tau)= & \frac{1}{2} \sigma^{2}\left(h_{1}^{2}+\frac{2 \eta_{1}}{\sigma^{2}} h_{1}+\frac{1}{\sigma^{2}} i x(i x+1)\right) \\
= & \frac{1}{2} \sigma^{2}\left(h_{1}+\frac{2 \eta_{1}+\sqrt{4 \eta_{1}^{2}-4 \sigma^{2} i x(i x+1)}}{2 \sigma^{2}}\right) \\
& \times\left(h_{1}+\frac{2 \eta_{1}-\sqrt{4 \eta_{1}^{2}-4 \sigma^{2} i x(i x+1)}}{2 \sigma^{2}}\right) \\
= & \frac{1}{2} \sigma^{2}\left(h_{1}+\frac{\eta_{1}+\Delta_{1}}{\sigma^{2}}\right)\left(h_{1}+\frac{\eta_{1}-\Delta_{1}}{\sigma^{2}}\right)
\end{aligned}
$$

where $\Delta_{1}=\sqrt{\eta_{1}^{2}-\sigma^{2} i x(i x+1)}$.

By the method of variable separation, we have

$$
\frac{2 \mathrm{~d} h_{1}}{\left(h_{1}+\frac{\eta_{1}+\Delta_{1}}{\sigma^{2}}\right)\left(h_{1}+\frac{\eta_{1}-\Delta_{1}}{\sigma^{2}}\right)}=\sigma^{2} \mathrm{~d} \tau .
$$


Using partial fractions, we get

$$
\frac{1}{\Delta_{1}}\left(\frac{1}{h_{1}+\frac{\eta_{1}-\Delta_{1}}{\sigma^{2}}}-\frac{1}{h_{1}+\frac{\eta_{1}+\Delta_{1}}{\sigma^{2}}}\right) \mathrm{d} h_{1}=\mathrm{d} \tau .
$$

Integrating both sides, we obtain

$$
\ln \left(\frac{h_{1}+\frac{\eta_{1}-\Delta_{1}}{\sigma^{2}}}{h_{1}+\frac{\eta_{1}+\Delta_{1}}{\sigma^{2}}}\right)=\Delta_{1} \tau+C .
$$

Using boundary condition $h_{1}(\tau=0)=0$, we get

$$
C=\ln \left(\frac{\eta_{1}-\Delta_{1}}{\eta_{1}+\Delta_{1}}\right) .
$$

Solving for $h_{1}$, we obtain

$$
h_{1}(\tau)=\frac{\left(\eta_{1}^{2}-\Delta_{1}^{2}\right)\left(e^{\Delta_{1} \tau}-1\right)}{\sigma^{2}\left(\eta_{1}+\Delta_{1}-\left(\eta_{1}-\Delta_{1}\right) e^{\Delta_{1} \tau}\right)} .
$$

In order to solve $g_{1}(\tau)$ explicitly, we substitute $h_{1}(\tau)$ into (20) and integrate with respect to $\tau$ on both sides. Then we get

$$
\begin{aligned}
g_{1}(\tau)= & \left(\left(r-\lambda^{S} m\right) i x-\lambda^{S} m\right) \tau \\
& -\frac{\kappa \theta}{\sigma^{2}}\left(2 \ln \left(1-\frac{\left(\Delta_{1}+\eta_{1}\right)\left(1-e^{-\Delta_{1} \tau}\right)}{2 \Delta_{1}}\right)+\left(\Delta_{1}+\eta_{1}\right) \tau\right) \\
& +\lambda^{S} \tau \int_{-\infty}^{\infty}\left(e^{(i x+1) y}-1\right) \phi_{Y}(y) \mathrm{d} y \\
& +\lambda^{v} \tau \int_{-\infty}^{\infty}\left(e^{z h_{1}(\tau)}-1\right) \phi_{Z}(z) \mathrm{d} z
\end{aligned}
$$

Proof of 2). The details of the proof are similar to case 1). Hence, we have

$$
f_{2}(l, v, t ; x, t+\tau)=\exp \left(g_{2}(\tau)+v h_{2}(\tau)+i x l+r \tau\right)
$$

where $g_{2}(\tau), h_{2}(\tau), \eta_{2}$ and $\Delta_{2}$ are as given in the Lemma.

We can thus evaluate the characteristic functions in explicit form. However, we are interested in the risk-neutral probabilities $\tilde{P}_{j}, j=1,2$. These can be inverted from the characteristic functions by performing the following integration

$$
\tilde{P}_{j}(l, v, t ; k, T)=\frac{1}{2}+\frac{1}{\pi} \int_{0^{+}}^{+\infty} \operatorname{Re}\left[\frac{e^{-i x k} f_{j}(l, v, t ; k, T)}{i x}\right] \mathrm{d} x
$$

for $j=1,2$.

To verify (21), firstly we note that

$$
\begin{aligned}
& E_{\mathcal{M}}\left[e^{i x\left(\ln S_{t}-\ln K\right)} \mid \ln S_{t}=L_{t}, v_{t}=v\right] \\
= & E_{\mathcal{M}}\left[e^{i x\left(L_{t}-k\right)} \mid L_{t}=l, v_{t}=v\right]=\int_{-\infty}^{\infty} e^{i x(l-k)} \mathrm{d} \tilde{P}_{j}(l, v, t ; k, T) \\
= & e^{-i x k} \int_{-\infty}^{\infty} e^{i x l} \mathrm{~d} \tilde{P}_{j}(l, v, t ; k, T)=e^{-i x k} \int_{-\infty}^{\infty} e^{i x k}(-\delta(l-k) \mathrm{d} k) \\
= & e^{-i x k} f_{j}(l, v, t ; k, T) .
\end{aligned}
$$

Then

$$
\begin{aligned}
& \frac{1}{2}+\frac{1}{\pi} \int_{0^{+}}^{+\infty} \operatorname{Re}\left[\frac{e^{-i x k} f_{j}(l, v, t ; k, T)}{i x}\right] \mathrm{d} x \\
= & \frac{1}{2}+\frac{1}{\pi} \int_{0^{+}}^{+\infty} \operatorname{Re}\left[\frac{E_{\mathcal{M}}\left[e^{i x\left(\ln S_{t}-\ln K\right)} \mid \ln S_{t}=L_{t}, v_{t}=v\right]}{i x}\right] \mathrm{d} x \\
= & E_{\mathcal{M}}\left[\frac{1}{2}+\frac{1}{\pi} \int_{0^{+}}^{+\infty} \operatorname{Re}\left[\frac{e^{i x(l-k)}}{i x}\right] \mathrm{d} x \mid L_{t}=l, v_{t}=v\right] \\
= & E_{\mathcal{M}}\left[\frac{1}{2}+\frac{1}{\pi} \int_{0^{+}}^{+\infty} \frac{\sin (x(l-k))}{x} \mathrm{~d} x \mid L_{t}=l, v_{t}=v\right] \\
= & E_{\mathcal{M}}\left[\frac{1}{2}+\operatorname{sgn}(l-k) \frac{1}{\pi} \int_{0^{+}}^{+\infty} \frac{\sin x}{x} \mathrm{~d} x \mid L_{t}=l, v_{t}=v\right] \\
= & E_{\mathcal{M}}\left[\frac{1}{2}+\frac{1}{2} \operatorname{sgn}(l-k) \mid L_{t}=l, v_{t}=v\right] \\
= & E_{\mathcal{M}}\left[1_{l \geq k} \mid L_{t}=l, v_{t}=v\right]
\end{aligned}
$$

where we have used the Dirichlet formula $\int_{0^{+}}^{+\infty} \frac{\sin x}{x} \mathrm{~d} x=1$ and the sgn function is defined as $\operatorname{sgn}(x)=1$ if $x>0$, 0 if $x=0$ and -1 if $x<0$. 